\title{
A Note on the Representative Adaptive Learning Algorithm
}

\section{Working Paper}

\section{Author(s):}

Bernardi, Michele; Galimberti, Jaqueson K.

Publication date:

2014-04

Permanent link:

https://doi.org/10.3929/ethz-a-010131559

Rights / license:

In Copyright - Non-Commercial Use Permitted

Originally published in:

KOF Working Papers 356 


\section{KOF Working Papers}

A Note on the Representative Adaptive Learning Algorithm

Michele Berardi and Jaqueson K. Galimberti 


\section{KOF}

ETH Zurich

KOF Swiss Economic Institute WEH D 4

Weinbergstrasse 35

8092 Zurich

Switzerland

Phone +41446324239

Fax +41446321218

www.kof.ethz.ch

kof@kof.ethz.ch 


\title{
A note on the representative adaptive learning algorithm*
}

\author{
MICHELE BERARDI \\ Economics, School of Social Sciences \\ University of Manchester
}

\author{
JAQUESON K. GaLIMBERTI \\ KOF Swiss Economic Institute \\ ETH Zurich
}

\begin{abstract}
We compare forecasts from different adaptive learning algorithms and calibrations applied to US real-time data on inflation and growth. We find that the Least Squares with constant gains adjusted to match (past) survey forecasts provides the best overall performance both in terms of forecasting accuracy and in matching (future) survey forecasts.

Keywords: expectations, learning algorithms, forecasting, learning-to-forecast, least squares, stochastic gradient.

JEL: C53, D83, D84, E03, E37.
\end{abstract}

\section{Introduction}

Adaptive learning algorithms have been proposed to provide an alternative to, and a justification for, rational expectations (RE) equilibria in macroeconomics (Evans and Honkapohja, 2001). Going beyond the RE hypothesis, however, comes at the cost of introducing another degree of freedom in macroeconomic modeling, since one has to be specific about which algorithm is assumed to represent agents behavior.

The usual choice for this purpose has been the Least Squares (LS) algorithm (Branch and Evans, 2006; Markiewicz and Pick, 2014), possibly due to its widespread popularity between econometricians. A computationally simpler alternative is offered by the Stochastic Gradient (SG) algorithm (Barucci and Landi, 1997; Evans and Honkapohja, 1998). We argue that the

\footnotetext{
${ }^{*}$ Accepted for publication in Economics Letters. A longer version of this paper has been previously circulated as "On the plausibility of adaptive learning in macroeconomics" (CGBCR Discussion Paper series, 177), which was presented at the 21th Symposium of the Society for Nonlinear Dynamics and Econometrics in Milan, 2013, and seminar series at: the University of Manchester, Lund University, and the KOF Swiss Economic Institute. We thank for useful comments provided by the participants of these events and by an anonymous referee.

†Corresponding author. E-mail: galimberti@kof.ethz.ch.
} 
previous literature has neglected the need of a realistic justification in the choice of the representative learning algorithm.

Importantly, theoretical analyses of learning convergence have shown that these learning algorithms may lead to different learnability conditions of RE equilibria (Heinemann, 2000; Giannitsarou, 2005). The LS dominance also has been challenged in previous applied studies (see Bullard and Eusepi, 2005; Carceles-Poveda and Giannitsarou, 2007). Hence, it remains open the question of which algorithm should be taken as representative from an empirical standpoint. Our main contribution is therefore an attempt to fill that gap, and we do this by comparing the quality and fit to surveys of the forecasts associated to each of these learning algorithms.

We estimate Vector Autoregressions (VARs) recursively with real-time quarterly data on US inflation and output growth, and then compare the associated multi-horizon forecasts over an evaluation sample from 1981q1 to 2011q4. Details of our approach are presented in section 2. Our results, presented in section 3, are favorable to the use of the LS as representative of agents learning-to-forecast behavior for the growth variable, whereas for inflation we obtained mixed evidence depending on the calibration of the learning gains. Namely, the LS dominance is weakened when the learning gains are calibrated so as to minimize observed squared forecasting errors rather than their distance to survey forecasts. We discuss these results in section 4.

\section{Approach}

Our approach is based on learning-to-forecast exercises that mimic the real-time environment faced by an economic agent when forming expectations on inflation $\left(\pi_{t}\right)$ and output growth $\left(g_{t}\right)$. We assume this agent attempts to construct inferences about these variables estimating a VAR of the form

$$
y_{i, t}=\mathbf{x}_{t}^{\prime} \boldsymbol{\theta}_{i, t}+\varepsilon_{i, t}
$$

where $y_{1, t}=\pi_{t}$ and $y_{2, t}=g_{t}, \mathbf{x}_{t}=\left(1, \pi_{t-1}, \ldots, \pi_{t-p}, g_{t-1}, \ldots, g_{t-p}\right)^{\prime}, \boldsymbol{\theta}_{i, t}=\left(\theta_{0, i, t}, \theta_{1, i, t}, \ldots, \theta_{p, i, t}\right.$, $\left.\theta_{p+1, i, t}, \ldots, \theta_{2 p, i, t}\right)^{\prime}, p$ denotes the VAR lag order, and $\varepsilon_{i, t}$ is a white noise disturbance. To estimate each equation's vector of coefficients, $\boldsymbol{\theta}_{i, t}$, we follow the adaptive learning literature and adopt the LS and the SG specifications. 
Algorithm 1 (LS). Under the estimation context of (1), the LS algorithm assumes the form of

$$
\begin{aligned}
\hat{\boldsymbol{\theta}}_{i, t}^{L S} & =\hat{\boldsymbol{\theta}}_{i, t-1}^{L S}+\gamma_{t} \mathbf{R}_{t}^{-1} \mathbf{x}_{t}\left(y_{i, t}-\mathbf{x}_{t}^{\prime} \hat{\boldsymbol{\theta}}_{i, t-1}^{L S}\right), \\
\mathbf{R}_{t} & =\mathbf{R}_{t-1}+\gamma_{t}\left(\mathbf{x}_{t} \mathbf{x}_{t}^{\prime}-\mathbf{R}_{t-1}\right),
\end{aligned}
$$

where $\gamma_{t}$ is a learning gain parameter, and $\mathbf{R}_{t}$ stands for an estimate of regressors matrix of second moments, $E\left[\mathbf{x}_{t} \mathbf{x}_{t}^{\prime}\right]$.

Algorithm 2 (SG). Under the estimation context of (1), the SG algorithm is given by

$$
\hat{\boldsymbol{\theta}}_{i, t}^{S G}=\hat{\boldsymbol{\theta}}_{i, t-1}^{S G}+\mu_{t} \mathbf{x}_{t}\left(y_{i, t}-\mathbf{x}_{t}^{\prime} \hat{\boldsymbol{\theta}}_{i, t-1}^{S G}\right)
$$

with $\mu_{t}$ standing for the learning gain parameter.

We then use the LS and the SG algorithms to obtain recursive estimates of the parameters of VAR model specifications ( 1 to 4 lag orders) applied to real-time quarterly data on US real GNP/GDP and its price index from 1947q2 to 2011q4. Our data on these series comes from the Philadelphia's Fed Real-Time Data Research Center and consists of vintages from 1966q1 to $2012 q 1$, i.e., a total of 185 snapshots of what was known on these variables by a market participant in real-time (see Stark and Croushore, 2002). For the purpose of comparing the algorithms forecasts to those provided by survey respondents, we use data from the Survey of Professional Forecasters (SPF). Here we use the median of the individual forecasts made for a total of five horizons, namely from $t$ (nowcast) to $t+4$. The SPF data is available from $1968 q 4$ onwards, and, consistent to our data on actuals, the last survey data we use is that of $2010 q 4$, which contains forecasts up to 2011 q4.

Operation of these algorithms requires the specification of a (sequence of) gain value(s) determining how quickly some given information is incorporated into the algorithm's coefficients estimates. Recognizing the prominent role that the learning gains have in determining the statistical properties of the estimates associated to each algorithm (see, e.g., Benveniste et al., 1990), here we follow the calibration approach proposed in Berardi and Galimberti (2014). Particularly, we distinguish between two gain determination rationales: as a choice of rational agents, selecting the gains that minimize the (average) squared forecasting errors over a given window of observations; and as a primitive parameter of agents behavior, where the gains are selected so as to minimize the distance of the algorithms forecasts to those collected through 
survey forecasts.

Regarding the windows used to select the gains according to the criteria above we adopt two alternatives: a fixed and a time-varying gain calibration. Under the fixed calibration we pick the gain evaluating the corresponding criterion over the full sample of forecasts that we have computed, and keep this gain fixed throughout our exercise. For the time-varying calibration, in contrast, we use a rolling window sample of 60 forecasts to evaluate each gain determination criterion, hence selecting a new gain for every iteration on the real-time learning process. In both cases, the set of admissible gains is based on a grid of 100 values constructed taking an upper bound, experimentally computed to ensure algorithms stability, as reference.

Our design unfolds into a three-stages routine to generate the forecasts associated to each learning algorithm: initialization, estimation and forecasting, and evaluation. The first 75 observations in our sample (up to 1965q4) are used for the smoothing-based initialization of the algorithms (following Berardi and Galimberti, 2012). The next 60 observations (from 1966q1 to 1980q4) are used for the algorithms (first) time-varying calibration. Therefore, our evaluation sample corresponds to the period from $1981 \mathrm{q} 1$ to $2010 \mathrm{q} 4$. To match the timing of information in the SPF dataset, we compute and evaluate forecasts over five horizons, each of these with its own instance of gain calibrations.

\section{Results}

We start looking over the forecasts associated to each algorithm and gain value included in the grid computations. Figure 1 presents surfaces of average past performance for each algorithm and variable, showing their evolution through time and for the different gain values. Two main observations arise: (i) the behavior of each algorithm depends on the variable being forecasted, whereas for a given variable the LS and SG algorithms behave differently; (ii) the magnitudes of forecast errors were relatively higher during the first decade in our sample, irrespective of the variable forecasted and the algorithm used, an observation that can be associated with the period of greater volatility that preceded the Great Moderation in the US economy (see Stock and Watson, 2003). 
Figure 1: Evolution of algorithms' forecasting accuracy through time.
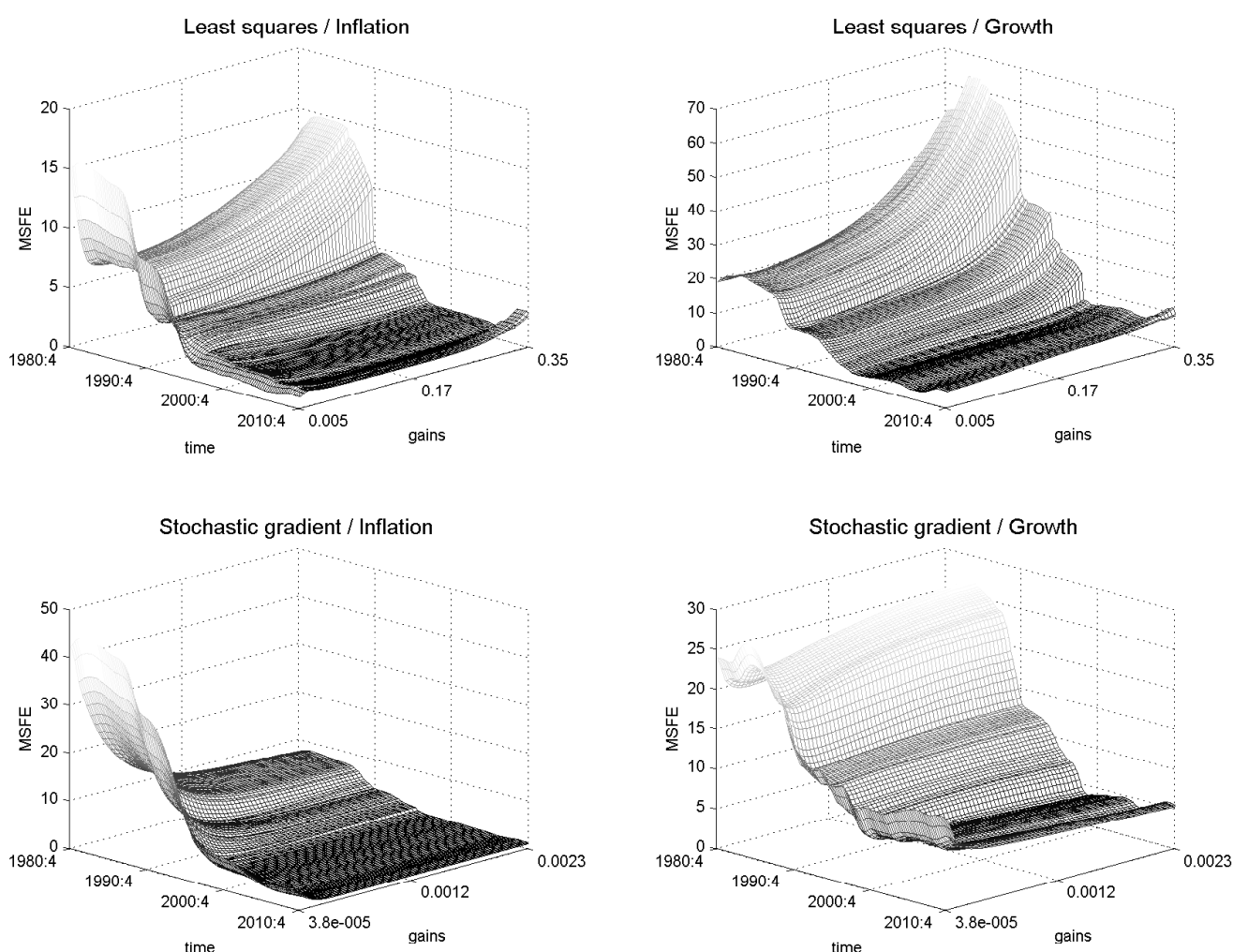

The MSFE plotted are computed on the basis of the 60 quarters backwards to the dates indicated into the NW-SE axis. The gain calibrations used for each algorithm are indicated into the SW-NE axis. Forecast errors refer to averaged errors over 5 horizons of forecasts.

To obtain a relative assessment of the learning mechanisms we now conduct two evaluation exercises comparing the forecasts associated to the learning algorithms with respect to their accuracy and their resemblance to the survey forecasts. We check for the statistical significance of these paired comparisons using tests common to the literature on forecast evaluation: the Diebold and Mariano (1995) (DM) test for equal (unconditional) predictive ability, and its more recently developed conditional counterpart test of Giacomini and White (2006) (GW). Our coverage of multiple forecasting horizons and VAR lag order specifications, for robustness, requires performing a high quantity of such comparisons ${ }^{1}$. Hence, to synthesize these evaluations we adopt hit rate measures, which are defined as the frequency by which the forecasts associated to a given algorithm is found to outperform those associate to its competitor with respect to one of our evaluation criteria.

\footnotetext{
${ }^{1}$ To be specific, 40 for each pair of algorithms/calibrations: 5 horizons $\times 4$ VARs $\times 2$ evaluation criteria. An Appendix is provided with the individual comparison results, and some descriptive statistics for each series of forecasts.
} 
We present in Tables 1 and 2 the hit rates for the comparisons between the LS and the SG algorithms ${ }^{2}$. In terms of accuracy a clear dominance of the LS is observed in forecasting growth. For inflation, the results depend on the approach adopted to the determination of the gains: while the SG is preferred under the gain as a choice, the LS tends to outperform the SG when the gain is taken as a primitive. Notice, however, that the tests for equal predictive ability rarely find statistical significance for the LS victories in forecasting inflation. Hence, our reading of these accuracy results is that they represent mixed evidence in support of each of the algorithms.

Table 1: Hit rates comparing the algorithms forecast accuracy.

\begin{tabular}{|c|c|c|c|c|c|c|}
\hline \multirow{2}{*}{$\begin{array}{l}\text { Variables } \\
\text { - Gains }\end{array}$} & \multicolumn{3}{|c|}{ LS wins } & \multicolumn{3}{|c|}{ SG wins } \\
\hline & Hit rate & DM-20\% & GW-20\% & Hit rate & DM-20\% & GW-20\% \\
\hline \multicolumn{7}{|c|}{ Inflation, gains as a choice } \\
\hline - Fixed & $20 \%$ & $0 \%$ & $0 \%$ & $80 \%$ & $25 \%$ & $20 \%$ \\
\hline - Time-varying & $35 \%$ & $0 \%$ & $0 \%$ & $65 \%$ & $20 \%$ & $20 \%$ \\
\hline \multicolumn{7}{|c|}{ Inflation, gains as a primitive } \\
\hline - Fixed & $65 \%$ & $0 \%$ & $0 \%$ & $35 \%$ & $15 \%$ & $15 \%$ \\
\hline - Time-varying & $60 \%$ & $0 \%$ & $5 \%$ & $40 \%$ & $15 \%$ & $20 \%$ \\
\hline \multicolumn{7}{|c|}{ Growth, gains as a choice } \\
\hline - Fixed & $95 \%$ & $75 \%$ & $10 \%$ & $5 \%$ & $0 \%$ & $5 \%$ \\
\hline - Time-varying & $75 \%$ & $30 \%$ & $40 \%$ & $25 \%$ & $0 \%$ & $5 \%$ \\
\hline \multicolumn{7}{|c|}{ Growth, gains as a primitive } \\
\hline - Fixed & $100 \%$ & $70 \%$ & $10 \%$ & $0 \%$ & $0 \%$ & $0 \%$ \\
\hline - Time-varying & $100 \%$ & $55 \%$ & $20 \%$ & $0 \%$ & $0 \%$ & $0 \%$ \\
\hline
\end{tabular}

The hit rates sum up forecasting horizons (5) and VAR lag orders (4). DM-20\% and GW-20\% represents the frequency within which the associated specification was found to have superior performance with statistical significance below the $20 \%$ level using the DM and the GW tests.

\footnotetext{
${ }^{2}$ We adopt a $20 \%$ level of significance as reference due to the low power of the tests to detect statistical differences between the algorithms series of forecasts. Under a $10 \%$ level of significance (see the Appendix) we observe a systematic decrease in these hit rates, meaning that our relative conclusions remain qualitatively unchanged.
} 
Table 2: Hit rates comparing the algorithms forecast resemblance to surveys.

\begin{tabular}{|c|c|c|c|c|c|c|}
\hline \multirow{2}{*}{$\begin{array}{l}\text { Variables } \\
\text { - Calibrations }\end{array}$} & \multicolumn{3}{|c|}{ LS wins } & \multicolumn{3}{|c|}{ SG wins } \\
\hline & Hit rate & DM-20\% & GW-20\% & Hit rate & DM-20\% & GW-20\% \\
\hline \multicolumn{7}{|c|}{ Inflation, gains as a choice } \\
\hline - Fixed & $80 \%$ & $45 \%$ & $30 \%$ & $20 \%$ & $5 \%$ & $5 \%$ \\
\hline - Time-varying & $100 \%$ & $75 \%$ & $35 \%$ & $0 \%$ & $0 \%$ & $0 \%$ \\
\hline \multicolumn{7}{|c|}{ Inflation, gains as a primitive } \\
\hline - Fixed & $95 \%$ & $55 \%$ & $40 \%$ & $5 \%$ & $0 \%$ & $0 \%$ \\
\hline - Time-varying & $100 \%$ & $75 \%$ & $40 \%$ & $0 \%$ & $0 \%$ & $0 \%$ \\
\hline \multicolumn{7}{|c|}{ Growth, gains as a choice } \\
\hline - Fixed & $75 \%$ & $70 \%$ & $65 \%$ & $25 \%$ & $5 \%$ & $20 \%$ \\
\hline - Time-varying & $65 \%$ & $40 \%$ & $65 \%$ & $35 \%$ & $5 \%$ & $5 \%$ \\
\hline \multicolumn{7}{|c|}{ Growth, gains as a primitive } \\
\hline - Fixed & $80 \%$ & $70 \%$ & $65 \%$ & $20 \%$ & $0 \%$ & $20 \%$ \\
\hline - Time-varying & $75 \%$ & $65 \%$ & $75 \%$ & $25 \%$ & $0 \%$ & $10 \%$ \\
\hline
\end{tabular}

The results on the forecasts resemblance to surveys, in contrast, indicate an overwhelming dominance of the LS algorithm. Other than beating the SG resemblance with a higher frequency, the victories of the LS are often found with statistical significance by the tests of equal predictive ability. Also notice that, if the SG presents any threat to the LS dominance, this would not be on the grounds of their resemblance to inflation but to the growth survey forecasts. Clearly, this is a disturbing observation given that in our previous exercise the slightly favorable evidence for the SG was observed in forecasting inflation, but not for growth.

\section{Discussion}

Our results on the algorithms forecasting performance suggest that their relative suitability depends mainly on the statistical properties of the data environment to which they are applied. Compared to inflation rates, output growth is known to have a lower degree of dynamic persistence and a higher degree of volatility, which makes of growth a variable harder to forecast than inflation (see, e.g., Patton and Timmermann, 2011). Hence, it seems reasonable to find that the more "sophisticated" LS method was favored in forecasting growth.

But it is instructive to see that, in some cases, the simpler SG was able to outperform the LS in forecasting inflation. These results for inflation also seem to be consistent to the recent findings of Faust and Wright (2013) showing that simpler forecasting methods tend to outperform more complicated model-based forecasts of inflation. Taking the surveys as 
a reference, nevertheless, pointed to an overall dominance of the LS in providing forecasts closer to those representing actual agents forecasts. Clearly, this result provides support for the usually unquestioned choice of the LS learning scheme in the applied literature on adaptive learning in macroeconomics.

A key aspect to understand our mixed findings relates to the two alternative approaches to the determination of the gains we evaluated. In a companion paper (Berardi and Galimberti, 2014) we have motivated these two gain determination perspectives as a choice of rationally optimizing agents, and as a primitive parameter of bounded rational agents, respectively, finding evidence favorable to the latter. Given that under the primitive determination of the gains our results were overall in favor to the LS, we conclude that our empirical evidence provides support for the use of this algorithm as representative of agents learning-to-forecast behavior.

Nevertheless, our results suffer from the lack of a direct economic interpretation due to our focus on non structural model specifications. We leave this as a motivation for future research on the issue of the representative learning algorithm.

\section{Appendix}

\section{A Details on data}

Short time series history: some vintages lack of earlier observations due to delays into BEA revisions (see Philadelphia's Fed documentations). This was the case of the vintages of 1992q1-1992q4 (missing data from 1947-1958), 1996q1-1997q1 (missing data from 1947-1959q2), and 1999q4-2000q1 (missing data from 1947-1958). We circumvent this problem (to turn the dataset vintages-balanced) by reproducing observations from the last available vintage while rescaling in accordance to the ratio between the first observation available in the missing observation vintage and the value observed for the same period in the vintage being used as source for the missing observations.

Missing observation for 1995q4 in vintage 1996q1: as a result of the US federal government shutdown in late 1995, the observation for $1995 q 4$ was missing in the $1996 q 1$ vintage. Fortunately, this is the only point in this dataset that this happens. We fulfill this gap by using the observation available in the March 1996 monthly vintage 
for the same series. Incidentally, the SPF 1996q1 median backcast for $1995 q 4$ is identical to the value later observed in March 1996, thence, our simplifying procedure is not favoring any method.

Caveat on SPF's forecasts for Real GDP: forecasts for real GDP were not asked in the surveys prior to 1981q3. To extend this series of forecast back to 1968q4, real GDP prior to $1981 \mathrm{q} 3$ is computed by using the formula (nominal GDP / GDP prices) * 100.

\section{B Review of statistical tests for equal predictive ability}

We want to determine whether two series of forecasts are statistically different from each other. Let $f_{1, t, h}$ and $f_{2, t, h}$ stand for these forecasts, where $h$ (going from 0 to 4 in our case) denotes the horizon at which these forecasts were made, and $y_{t}$ stand for the series of targets of these forecasts. Let the losses associated to each of these forecasts be given by $\mathcal{L}\left(f_{1, t, h}, y_{t}\right)$ and $\mathcal{L}\left(f_{2, t, h}, y_{t}\right)$. Letting $d_{t, h}=\mathcal{L}\left(f_{1, t, h}, y_{t}\right)-\mathcal{L}\left(f_{2, t, h}, y_{t}\right)$ denote the series of loss differentials between the two forecasts at horizon $h$, the Diebold and Mariano (1995) test evaluates whether their average loss differences,

$$
\bar{d}_{h}=\frac{1}{T} \sum_{i=1}^{T} d_{i, h},
$$

is significantly different from zero. Under the null hypothesis of equal predictive ability the DM statistic,

$$
D M_{h}=\frac{\bar{d}_{h}}{\sqrt{\hat{\sigma}_{d}^{2} / T}},
$$

has a $t$-distribution with $T-1$ degrees of freedom, where $\hat{\sigma}_{d}^{2}$ is an estimate of the long-run variance of $d_{t, h}$. For the estimation of $\hat{\sigma}_{d}^{2}$ we adopt the heteroskedasticity and autocorrelation consistent (HAC) estimator proposed by Newey and West (1987).

The Giacomini and White (2006) test, in contrast, evaluates the null hypothesis of equal conditional predictive ability. The main caveat on this test relates to the specification of a test function, $\mathbf{q}_{t, h}$ containing $q$ instruments, which attempts to control for the informational conditioning required by the null hypothesis. To test the conditional moment restriction $E\left[\mathbf{q}_{t, h} d_{t, h}\right]=\mathbf{0}$, 
a Wald-type test statistic is proposed having the form of

$$
G W_{h}=T\left(T^{-1} \sum_{i=1}^{T} \mathbf{q}_{i, h} d_{i, h}\right)^{\prime} \hat{\boldsymbol{\Omega}}_{h}^{-1}\left(T^{-1} \sum_{i=1}^{T} \mathbf{q}_{i, h} d_{i, h}\right)
$$

where $\hat{\boldsymbol{\Omega}}_{h}$ is a $q \times q$ consistent estimate of the covariance matrix of $\mathbf{q}_{t, h} d_{t, h}$. Under the null hypothesis of equal conditional predictive ability $G W_{h}$ has a $\chi_{q}^{2}$ distribution.

Apart from the first horizon, $\hat{\Omega}_{h}$ is again estimated using the HAC estimator of Newey and West (1987), with $h$ determining the truncated kernel bandwidth. For the case of the first horizon, Giacomini and White (2006) simplify the computation of (7) to be given by $T R^{2}$, where $R^{2}$ is the uncentered squared multiple correlation coefficient obtained by regressing a constant unity on $\mathbf{q}_{t, h} d_{t, h}$. Finally, regarding the specification of $\mathbf{q}_{t, h}$, in the lack of better alternatives, the recommendation is for the use of $h$-lagged loss differentials. Thus, in our calculations we set $\mathbf{q}_{t, h}=d_{t-h, h}$.

\section{Supplementary statistics}

In table 3 we present statistics for each individual series of forecasts. Some observations sprout from these statistics: (i) the LS (SG) forecasts tend to be biased up(down)wards; (ii) the forecasts fail to replicate growth rates variability, whereas for inflation the SG forecasts presented variances closer to that of the actuals; (iii) between each algorithm's calibrations there is little variation in terms of their forecasts statistical properties. 
Table 3: Data and forecasts statistics by algorithm/calibration.

(a) Inflation.

\begin{tabular}{lccccccccc}
\hline Series/Algorithm & Mean & Min & Max & Var & AR(1) & CorA & CorS & MSFE & MSFCE \\
\hline Actuals & 2.67 & -0.33 & 9.39 & 2.68 & 0.63 & 1.00 & 0.81 & 0.00 & 1.03 \\
Surveys & 2.93 & 0.62 & 9.49 & 2.46 & 0.94 & 0.81 & 1.00 & 1.03 & 0.00 \\
Least Squares & & & & & & & & & \\
-Fixed /choice & 2.99 & -0.14 & 10.30 & 2.94 & 0.83 & 0.73 & 0.90 & 1.62 & 0.55 \\
-Time-var./choice & 3.07 & 0.13 & 10.35 & 2.80 & 0.81 & 0.72 & 0.90 & 1.69 & 0.56 \\
-Fixed/primit. & 2.97 & -0.34 & 10.32 & 2.75 & 0.74 & 0.70 & 0.90 & 1.71 & 0.54 \\
-Time-var./primit. & 3.06 & 0.01 & 10.39 & 2.57 & 0.78 & 0.72 & 0.89 & 1.62 & 0.55 \\
Stochastic Gradient & & & & & & & & & \\
-Fixed /choice & 2.77 & 0.12 & 11.89 & 2.65 & 0.69 & 0.67 & 0.88 & 1.76 & 0.62 \\
-Time-var./choice & 2.69 & -0.83 & 11.89 & 2.79 & 0.74 & 0.69 & 0.89 & 1.69 & 0.63 \\
-Fixed/primit. & 2.77 & 0.12 & 11.89 & 2.65 & 0.69 & 0.67 & 0.88 & 1.76 & 0.62 \\
-Time-var./primit. & 2.78 & -0.08 & 11.84 & 2.62 & 0.68 & 0.67 & 0.88 & 1.76 & 0.63 \\
\hline
\end{tabular}

(b) Growth.

\begin{tabular}{lccccccccc}
\hline Series/Algorithm & Mean & Min & Max & Var & AR(1) & CorA & CorS & MSFE & MSFCE \\
\hline Actuals & 2.54 & -6.14 & 8.67 & 6.17 & 0.50 & 1.00 & 0.78 & 0.00 & 2.56 \\
Surveys & 2.24 & -5.19 & 7.01 & 3.14 & 0.72 & 0.78 & 1.00 & 2.56 & 0.00 \\
Least Squares & & & & & & & & & \\
-Fixed /choice & 3.04 & -3.66 & 6.58 & 1.58 & 0.60 & 0.39 & 0.58 & 5.55 & 2.74 \\
-Time-var./choice & 3.08 & -0.95 & 6.70 & 1.18 & 0.53 & 0.38 & 0.63 & 5.55 & 2.61 \\
-Fixed/primit. & 3.01 & -2.02 & 5.46 & 1.07 & 0.58 & 0.47 & 0.64 & 5.03 & 2.46 \\
-Time-var./primit. & 2.98 & -0.95 & 6.00 & 1.10 & 0.52 & 0.42 & 0.66 & 5.24 & 2.34 \\
Stochastic Gradient & & & & & & & & & \\
-Fixed /choice & 1.88 & -2.13 & 4.66 & 1.32 & 0.48 & 0.43 & 0.59 & 5.44 & 2.16 \\
-Time-var./choice & 2.10 & -1.24 & 4.75 & 1.32 & 0.53 & 0.40 & 0.55 & 5.38 & 2.23 \\
-Fixed/primit. & 1.88 & -2.13 & 4.66 & 1.32 & 0.48 & 0.43 & 0.59 & 5.44 & 2.16 \\
-Time-var./primit. & 2.07 & -1.85 & 4.68 & 1.46 & 0.53 & 0.41 & 0.56 & 5.37 & 2.20 \\
\hline
\end{tabular}

The forecasts statistics refer to those obtained for the first forecasting horizon, $h=0$, and over the full evaluation sample from $1981 q 1$ to $2010 q 4$. The algorithms' forecasts refer to those from the $\operatorname{VAR}(1) . A R(1)$ stands for the first order autocorrelation of each series, CorA and CorS stands for the correlation with the series of actuals (first-available in real-time) and survey forecasts, respectively, and $M S F(C) E$ stands for the mean squared forecast (comparison) errors.

In tables 4-7 we present the individual comparisons of forecasting performance (accuracy and resemblance) between the LS and SG algorithms. These results represent the input for the hit rates presented in the main text. To illustrate that definition consider the LS accuracy hit rate under the fixed gain determined as a choice, forecasting inflation: that hit rate was found equal to $20 \%$, e.g., indicating that the LS algorithm outperformed the SG only in 4 out of the 20 comparisons conducted for each combination of VAR lag order and forecasting horizon. These cases can be easily identified from the first set of results in the upper left portion of 
table 4: the LS outperformed the SG for the $\operatorname{VAR}(1)$ at the first horizon, the $\operatorname{VAR}(2)$ at the first and the last horizon, and for the $\operatorname{VAR}(3)$ at the last horizon. The DM-20\% and GW-20\% statistics associated to these hit rates then represent the frequency by which the forecasting (comparison) errors associated to the outperforming method is found to be statistically different of its competitor according to the corresponding test at a $20 \%$ level of significance. So, in our example case for the LS/fixed/choice/inflation accuracy hit rates we have that none of the 4 cases where the LS outperformed the SG presented a DM/GW test $p$-value below $20 \%$, which is also evident from the results in table 4. 
Table 4: Comparitive evaluations of algorithms forecasting accuracy for inflation.

\begin{tabular}{|c|c|c|c|c|c|c|c|c|c|c|c|c|}
\hline \multirow{3}{*}{$\begin{array}{l}\text { Calibration } \\
\text { - Model } \\
\\
\text { - horizon }\end{array}$} & \multicolumn{6}{|c|}{ Gains as a choice } & \multicolumn{6}{|c|}{ Gains as a primitive } \\
\hline & \multicolumn{2}{|c|}{ MSFE } & \multicolumn{2}{|c|}{$\mathrm{DM}$} & \multicolumn{2}{|c|}{ GW } & \multicolumn{2}{|c|}{ MSFE } & \multicolumn{2}{|c|}{ DM } & \multicolumn{2}{|c|}{ GW } \\
\hline & LS & SG & Stat. & p-val. & Stat. & p-val. & LS & SG & Stat. & p-val. & Stat. & p-val. \\
\hline \multicolumn{13}{|l|}{ Fixed gains } \\
\hline - VAR(1)- $h=0$ & 1.62 & 1.76 & -0.78 & 0.44 & 0.00 & 0.98 & 1.71 & 1.76 & -0.40 & 0.69 & 0.26 & 0.61 \\
\hline$-h=1$ & 1.88 & 1.86 & 0.09 & 0.92 & 0.59 & 0.44 & 1.94 & 1.86 & 0.33 & 0.74 & 0.24 & 0.63 \\
\hline$-h=2$ & 1.81 & 1.22 & 1.31 & 0.19 & 1.62 & 0.20 & 1.75 & 1.22 & 2.20 & 0.03 & 0.18 & 0.67 \\
\hline$-h=3$ & 2.16 & 1.42 & 1.57 & 0.12 & 0.01 & 0.91 & 2.06 & 1.42 & 1.78 & 0.08 & 0.01 & 0.92 \\
\hline$-h=4$ & 2.60 & 2.01 & 1.02 & 0.31 & 0.00 & 0.94 & 2.55 & 2.01 & 0.88 & 0.38 & 4.99 & 0.03 \\
\hline$-\operatorname{VAR}(2)-h=0$ & 1.51 & 1.56 & -0.43 & 0.67 & 0.02 & 0.87 & 1.47 & 1.53 & -0.59 & 0.56 & 0.01 & 0.90 \\
\hline$-h=1$ & 1.72 & 1.69 & 0.14 & 0.89 & 0.01 & 0.91 & 1.62 & 1.66 & -0.25 & 0.80 & 0.02 & 0.90 \\
\hline$-h=2$ & 1.40 & 1.11 & 1.78 & 0.08 & 2.15 & 0.14 & 1.31 & 1.09 & 2.04 & 0.04 & 3.29 & 0.07 \\
\hline$-h=3$ & 1.64 & 1.62 & 0.06 & 0.95 & 0.00 & 0.96 & 1.56 & 1.48 & 0.27 & 0.79 & 0.45 & 0.50 \\
\hline$-h=4$ & 2.08 & 2.14 & -0.11 & 0.91 & 0.71 & 0.40 & 1.94 & 1.96 & -0.04 & 0.97 & 0.67 & 0.41 \\
\hline$-\operatorname{VAR}(3)-h=0$ & 1.37 & 1.34 & 0.27 & 0.79 & 0.00 & 0.96 & 1.34 & 1.34 & -0.02 & 0.98 & 0.36 & 0.55 \\
\hline$-h=1$ & 1.53 & 1.48 & 0.40 & 0.69 & 0.02 & 0.88 & 1.44 & 1.46 & -0.20 & 0.84 & 0.55 & 0.46 \\
\hline$-h=2$ & 1.43 & 1.22 & 1.64 & 0.10 & 1.65 & 0.20 & 1.24 & 1.21 & 0.28 & 0.78 & 1.73 & 0.19 \\
\hline$-h=3$ & 1.77 & 1.72 & 0.14 & 0.89 & 0.01 & 0.93 & 1.56 & 1.64 & -0.31 & 0.76 & 0.46 & 0.50 \\
\hline$-h=4$ & 2.10 & 2.32 & -0.37 & 0.71 & 0.77 & 0.38 & 1.86 & 2.11 & -0.54 & 0.59 & 0.62 & 0.43 \\
\hline$-\operatorname{VAR}(4)-h=0$ & 1.44 & 1.44 & 0.01 & 0.99 & 2.95 & 0.09 & 1.39 & 1.44 & -0.39 & 0.70 & 1.24 & 0.26 \\
\hline$-h=1$ & 1.71 & 1.54 & 1.03 & 0.31 & 0.06 & 0.81 & 1.52 & 1.52 & -0.02 & 0.98 & 0.34 & 0.56 \\
\hline$-h=2$ & 1.63 & 1.49 & 1.30 & 0.20 & 3.37 & 0.07 & 1.31 & 1.47 & -0.84 & 0.40 & 0.85 & 0.36 \\
\hline$-h=3$ & 1.99 & 1.91 & 0.23 & 0.82 & 0.06 & 0.80 & 1.60 & 1.88 & -0.77 & 0.44 & 0.66 & 0.42 \\
\hline$-h=4$ & 2.54 & 2.41 & 0.22 & 0.82 & 0.08 & 0.77 & 2.07 & 2.22 & -0.45 & 0.65 & 0.05 & 0.83 \\
\hline \multicolumn{13}{|c|}{ Time-varying gains } \\
\hline$-\operatorname{VAR}(1)-h=0$ & 1.69 & 1.69 & 0.02 & 0.99 & 0.90 & 0.34 & 1.62 & 1.76 & -0.80 & 0.42 & 0.98 & 0.32 \\
\hline$-h=1$ & 1.88 & 1.86 & 0.09 & 0.93 & 0.24 & 0.62 & 1.92 & 1.87 & 0.17 & 0.87 & 0.40 & 0.53 \\
\hline$-h=2$ & 1.97 & 1.26 & 1.69 & 0.09 & 1.70 & 0.19 & 1.70 & 1.23 & 1.91 & 0.06 & 0.45 & 0.50 \\
\hline$-h=3$ & 2.32 & 1.34 & 2.10 & 0.04 & 0.02 & 0.89 & 1.95 & 1.42 & 1.50 & 0.14 & 0.07 & 0.79 \\
\hline$-h=4$ & 2.74 & 1.95 & 1.26 & 0.21 & 0.16 & 0.69 & 2.25 & 2.01 & 0.41 & 0.68 & 3.54 & 0.06 \\
\hline$-\operatorname{VAR}(2)-\mathrm{h}=0$ & 1.57 & 1.54 & 0.28 & 0.78 & 0.01 & 0.92 & 1.48 & 1.53 & -0.53 & 0.60 & 0.01 & 0.93 \\
\hline$-h=1$ & 1.69 & 1.75 & -0.34 & 0.73 & 0.04 & 0.85 & 1.58 & 1.67 & -0.50 & 0.62 & 0.01 & 0.91 \\
\hline$-h=2$ & 1.41 & 1.15 & 1.41 & 0.16 & 1.79 & 0.18 & 1.25 & 1.09 & 1.47 & 0.14 & 3.62 & 0.06 \\
\hline$-h=3$ & 1.61 & 1.62 & -0.03 & 0.97 & 0.11 & 0.74 & 1.47 & 1.52 & -0.15 & 0.88 & 0.22 & 0.64 \\
\hline$-h=4$ & 1.99 & 2.34 & -0.48 & 0.64 & 0.94 & 0.33 & 1.79 & 2.10 & -0.53 & 0.60 & 0.73 & 0.39 \\
\hline$-\operatorname{VAR}(3)-h=0$ & 1.44 & 1.32 & 1.36 & 0.18 & 0.03 & 0.86 & 1.37 & 1.32 & 0.59 & 0.56 & 0.25 & 0.62 \\
\hline$-h=1$ & 1.56 & 1.52 & 0.28 & 0.78 & 0.89 & 0.35 & 1.45 & 1.48 & -0.27 & 0.79 & 0.36 & 0.55 \\
\hline$-h=2$ & 1.39 & 1.23 & 1.17 & 0.24 & 1.90 & 0.17 & 1.24 & 1.23 & 0.04 & 0.96 & 2.01 & 0.16 \\
\hline$-h=3$ & 1.70 & 1.75 & -0.14 & 0.89 & 0.00 & 0.99 & 1.49 & 1.69 & -0.65 & 0.51 & 0.30 & 0.59 \\
\hline$-h=4$ & 1.98 & 2.46 & -0.78 & 0.44 & 1.14 & 0.28 & 1.83 & 2.18 & -0.76 & 0.45 & 1.47 & 0.23 \\
\hline$-\operatorname{VAR}(4)-h=0$ & 1.50 & 1.43 & 0.51 & 0.61 & 0.44 & 0.51 & 1.47 & 1.42 & 0.41 & 0.68 & 2.15 & 0.14 \\
\hline$-h=1$ & 1.72 & 1.54 & 0.90 & 0.37 & 0.61 & 0.44 & 1.51 & 1.53 & -0.15 & 0.88 & 0.38 & 0.54 \\
\hline$-h=2$ & 1.61 & 1.53 & 0.63 & 0.53 & 4.28 & 0.04 & 1.36 & 1.54 & -1.10 & 0.28 & 2.45 & 0.12 \\
\hline$-h=3$ & 1.92 & 1.95 & -0.10 & 0.92 & 0.08 & 0.77 & 1.55 & 1.97 & -1.24 & 0.22 & 0.45 & 0.50 \\
\hline$-\mathrm{h}=4$ & 2.33 & 2.48 & -0.29 & 0.78 & 0.42 & 0.52 & 1.93 & 2.25 & -0.96 & 0.34 & 0.11 & 0.74 \\
\hline
\end{tabular}

We highlight in bold the algorithm presenting lower $\mathrm{MSF}(\mathrm{C}) \mathrm{E}$ by comparison. We do the same to highlight those comparisons for which statistically significant differences between the forecasts provided by each algorithm is found at levels below 20\%, using the Diebold and Mariano (1995) (DM) and the Giacomini and White (2006) (GW) tests. 
Table 5: Paired comparisons of algorithms forecasting accuracy for growth.

\begin{tabular}{|c|c|c|c|c|c|c|c|c|c|c|c|c|}
\hline \multirow{3}{*}{$\begin{array}{l}\text { Calibration } \\
\text { - Model } \\
\\
\quad \text { - horizon }\end{array}$} & \multicolumn{6}{|c|}{ Gains as a choice } & \multicolumn{6}{|c|}{ Gains as a primitive } \\
\hline & \multicolumn{2}{|c|}{ MSFE } & \multicolumn{2}{|c|}{$\mathrm{DM}$} & \multicolumn{2}{|c|}{ GW } & \multicolumn{2}{|c|}{ MSFE } & \multicolumn{2}{|c|}{ DM } & \multicolumn{2}{|c|}{ GW } \\
\hline & LS & $S G$ & Stat. & p-val. & Stat. & $\mathrm{p}$-val. & LS & SG & Stat. & p-val. & Stat. & p-val \\
\hline \multicolumn{13}{|l|}{ Fixed gains } \\
\hline$-\operatorname{VAR}(1)-h=0$ & 5.55 & 5.44 & 0.17 & 0.87 & 1.77 & 0.18 & 5.03 & 5.44 & -0.75 & 0.45 & 0.17 & 0.68 \\
\hline$-h=1$ & 6.65 & 7.24 & -0.64 & 0.53 & 0.21 & 0.64 & 6.20 & 7.18 & -1.17 & 0.25 & 0.04 & 0.84 \\
\hline$-h=2$ & 6.16 & 7.81 & -1.76 & 0.08 & 0.33 & 0.57 & 6.20 & 7.81 & -1.65 & 0.10 & 0.48 & 0.49 \\
\hline$-h=3$ & 6.16 & 8.16 & -1.95 & 0.05 & 0.78 & 0.38 & 6.28 & 7.38 & -0.99 & 0.32 & 1.03 & 0.31 \\
\hline$-\mathrm{h}=4$ & 5.57 & 7.82 & -1.96 & 0.05 & 0.04 & 0.85 & 5.69 & 7.02 & -1.13 & 0.26 & 0.67 & 0.41 \\
\hline$-\operatorname{VAR}(2)-\mathrm{h}=0$ & 5.05 & 5.50 & -1.23 & 0.22 & 0.44 & 0.51 & 4.90 & 5.48 & -1.88 & 0.06 & 1.00 & 0.32 \\
\hline$-h=1$ & 5.97 & 6.95 & -1.60 & 0.11 & 0.91 & 0.34 & 5.88 & 6.94 & -2.01 & 0.05 & 0.57 & 0.45 \\
\hline$-h=2$ & 6.00 & 7.65 & -2.17 & 0.03 & 0.19 & 0.67 & 6.00 & 7.60 & -2.22 & 0.03 & 0.25 & 0.62 \\
\hline$-h=3$ & 6.11 & 8.08 & -2.06 & 0.04 & 0.01 & 0.93 & 6.11 & 8.06 & -2.08 & 0.04 & 0.00 & 0.96 \\
\hline$-h=4$ & 5.64 & 7.87 & -2.03 & 0.04 & 0.46 & 0.50 & 5.64 & 7.84 & -1.99 & 0.05 & 0.13 & 0.72 \\
\hline$-\operatorname{VAR}(3)-h=0$ & 5.03 & 5.52 & -1.34 & 0.18 & 1.16 & 0.28 & 5.03 & 5.50 & -1.29 & 0.20 & 1.12 & 0.29 \\
\hline$-h=1$ & 5.96 & 7.00 & -1.84 & 0.07 & 2.16 & 0.14 & 5.96 & 6.93 & -1.82 & 0.07 & 2.41 & 0.12 \\
\hline$-h=2$ & 5.90 & 7.63 & -1.87 & 0.06 & 0.71 & 0.40 & 5.90 & 7.61 & -1.87 & 0.06 & 0.76 & 0.38 \\
\hline$-h=3$ & 6.00 & 8.01 & -1.77 & 0.08 & 0.01 & 0.93 & 6.00 & 8.06 & -1.78 & 0.08 & 0.00 & 0.97 \\
\hline$-h=4$ & 5.54 & 7.83 & -1.77 & 0.08 & 0.06 & 0.80 & 5.54 & 7.86 & -1.75 & 0.08 & 0.91 & 0.34 \\
\hline$-\operatorname{VAR}(4)-h=0$ & 5.30 & 5.72 & -0.93 & 0.36 & 0.74 & 0.39 & 5.30 & 5.70 & -0.88 & 0.38 & 0.69 & 0.40 \\
\hline$-h=1$ & 6.23 & 7.07 & -1.09 & 0.28 & 2.51 & 0.11 & 6.18 & 7.02 & -1.12 & 0.26 & 2.29 & 0.13 \\
\hline$-h=2$ & 6.18 & 7.66 & -1.39 & 0.17 & 0.63 & 0.43 & 6.18 & 7.67 & -1.42 & 0.16 & 0.71 & 0.40 \\
\hline$-h=3$ & 6.29 & 8.02 & -1.33 & 0.19 & 0.01 & 0.93 & 6.30 & 8.20 & -1.46 & 0.15 & 0.19 & 0.67 \\
\hline$-\mathrm{h}=4$ & 5.56 & 7.83 & -1.59 & 0.11 & 0.92 & 0.34 & 5.84 & 7.97 & -1.34 & 0.18 & 1.06 & 0.30 \\
\hline \multicolumn{13}{|c|}{ Time-varying gains } \\
\hline$-\operatorname{VAR}(1)-h=0$ & 5.55 & 5.38 & 0.30 & 0.76 & 2.92 & 0.09 & 5.24 & 5.37 & -0.28 & 0.78 & 3.67 & 0.06 \\
\hline$-h=1$ & 6.99 & 6.94 & 0.05 & 0.96 & 0.37 & 0.54 & 6.03 & 6.67 & -1.10 & 0.28 & 0.51 & 0.47 \\
\hline$-h=2$ & 7.25 & 7.37 & -0.09 & 0.93 & 2.33 & 0.13 & 6.16 & 7.18 & -1.32 & 0.19 & 0.93 & 0.34 \\
\hline$-h=3$ & 6.29 & 7.66 & -1.46 & 0.15 & 2.34 & 0.13 & 6.17 & 7.40 & -1.29 & 0.20 & 1.30 & 0.25 \\
\hline$-h=4$ & 5.76 & 7.12 & -1.31 & 0.19 & 0.09 & 0.76 & 5.62 & 6.98 & -1.24 & 0.22 & 0.27 & 0.60 \\
\hline$-\operatorname{VAR}(2)-\mathrm{h}=0$ & 5.72 & 5.66 & 0.12 & 0.91 & 0.01 & 0.91 & 4.93 & 5.52 & -1.84 & 0.07 & 0.57 & 0.45 \\
\hline$-h=1$ & 5.86 & 7.04 & -2.08 & 0.04 & 0.00 & 0.97 & 5.93 & 6.90 & -1.91 & 0.06 & 0.68 & 0.41 \\
\hline$-h=2$ & 7.59 & 7.76 & -0.09 & 0.93 & 1.92 & 0.17 & 6.00 & 7.50 & -2.19 & 0.03 & 0.07 & 0.79 \\
\hline$-h=3$ & 6.17 & 8.05 & -1.99 & 0.05 & 0.99 & 0.32 & 6.03 & 7.92 & -2.07 & 0.04 & 0.71 & 0.40 \\
\hline$-\mathrm{h}=4$ & 6.19 & 7.93 & -1.30 & 0.20 & 0.81 & 0.37 & 5.58 & 7.74 & -1.99 & 0.05 & 0.23 & 0.63 \\
\hline$-\operatorname{VAR}(3)-h=0$ & 5.77 & 5.74 & 0.09 & 0.93 & 1.26 & 0.26 & 5.33 & 5.66 & -0.64 & 0.52 & 1.28 & 0.26 \\
\hline$-h=1$ & 6.05 & 7.08 & -1.59 & 0.11 & 1.12 & 0.29 & 6.17 & 6.91 & -1.19 & 0.24 & 0.21 & 0.65 \\
\hline$-h=2$ & 6.95 & 7.77 & -0.54 & 0.59 & 1.81 & 0.18 & 5.98 & 7.57 & -1.80 & 0.07 & 0.40 & 0.52 \\
\hline$-h=3$ & 6.64 & 8.10 & -1.04 & 0.30 & 4.77 & 0.03 & 5.97 & 8.00 & -1.76 & 0.08 & 0.24 & 0.63 \\
\hline$-\mathrm{h}=4$ & 9.48 & 8.00 & 0.34 & 0.74 & 1.10 & 0.29 & 5.52 & 7.83 & -1.77 & 0.08 & 1.91 & 0.17 \\
\hline$-\operatorname{VAR}(4)-h=0$ & 5.72 & 5.83 & -0.33 & 0.75 & 0.21 & 0.65 & 5.50 & 5.70 & -0.45 & 0.66 & 0.90 & 0.34 \\
\hline$-h=1$ & 6.51 & 7.24 & -0.96 & 0.34 & 1.73 & 0.19 & 6.80 & 6.98 & -0.21 & 0.84 & 0.24 & 0.62 \\
\hline$-h=2$ & 7.07 & 7.91 & -0.54 & 0.59 & 1.86 & 0.17 & 7.00 & 7.81 & -0.51 & 0.61 & 1.35 & 0.24 \\
\hline$-h=3$ & 6.76 & 8.26 & -0.99 & 0.32 & 4.36 & 0.04 & 6.61 & 8.25 & -1.08 & 0.28 & 2.51 & 0.11 \\
\hline$-\mathrm{h}=4$ & 7.83 & 8.18 & -0.12 & 0.91 & 1.39 & 0.24 & 5.91 & 8.04 & -1.33 & 0.19 & 3.30 & 0.07 \\
\hline
\end{tabular}


Table 6: Paired comparisons of algorithms forecasting resemblance for inflation.

\begin{tabular}{|c|c|c|c|c|c|c|c|c|c|c|c|c|}
\hline \multirow{3}{*}{$\begin{array}{l}\text { Calibration } \\
\text { - Model } \\
\\
\text { - horizon }\end{array}$} & \multicolumn{6}{|c|}{ Gains as a choice } & \multicolumn{6}{|c|}{ Gains as a primitive } \\
\hline & \multicolumn{2}{|c|}{ MSFCE } & \multicolumn{2}{|c|}{ DM } & \multicolumn{2}{|c|}{ GW } & \multicolumn{2}{|c|}{ MSFCE } & \multicolumn{2}{|c|}{ DM } & \multicolumn{2}{|c|}{ GW } \\
\hline & LS & SG & Stat. & p-val. & Stat. & p-val. & LS & SG & Stat. & p-val. & Stat. & $\mathrm{p}$-val. \\
\hline \multicolumn{13}{|l|}{ Fixed gains } \\
\hline$-\operatorname{VAR}(1)-h=0$ & 0.55 & 0.62 & -0.93 & 0.36 & 1.85 & 0.17 & 0.54 & 0.62 & -1.04 & 0.30 & 2.11 & 0.15 \\
\hline$-h=1$ & 0.49 & 0.75 & -1.90 & 0.06 & 0.02 & 0.89 & 0.52 & 0.75 & -1.28 & 0.20 & 0.13 & 0.72 \\
\hline$-h=2$ & 0.49 & 0.79 & -1.73 & 0.09 & 2.21 & 0.14 & 0.38 & 0.79 & -1.60 & 0.11 & 3.09 & 0.08 \\
\hline$-h=3$ & 0.42 & 0.99 & -1.80 & 0.07 & 0.75 & 0.39 & 0.33 & 0.99 & -1.63 & 0.11 & 5.88 & 0.02 \\
\hline$-h=4$ & 0.41 & 1.21 & -1.65 & 0.10 & 1.87 & 0.17 & 0.49 & 1.21 & -1.42 & 0.16 & 2.13 & 0.14 \\
\hline$-\operatorname{VAR}(2)-\mathrm{h}=0$ & 0.45 & 0.44 & 0.18 & 0.86 & 2.30 & 0.13 & 0.38 & 0.42 & -0.89 & 0.37 & 1.54 & 0.21 \\
\hline$-h=1$ & 0.48 & 0.69 & -1.42 & 0.16 & 0.01 & 0.91 & 0.45 & 0.64 & -1.46 & 0.15 & 0.08 & 0.78 \\
\hline$-h=2$ & 0.36 & 0.82 & -1.94 & 0.05 & 3.07 & 0.08 & 0.33 & 0.68 & -1.92 & 0.06 & 1.63 & 0.20 \\
\hline$-h=3$ & 0.34 & 1.26 & -2.05 & 0.04 & 7.56 & 0.01 & 0.33 & 0.97 & -2.04 & 0.04 & 4.87 & 0.03 \\
\hline$-h=4$ & 0.44 & 1.39 & -2.06 & 0.04 & 1.87 & 0.17 & 0.46 & 1.21 & -2.14 & 0.03 & 1.68 & 0.20 \\
\hline$-\operatorname{VAR}(3)-h=0$ & 0.41 & 0.33 & 2.07 & 0.04 & 0.00 & 0.96 & 0.35 & 0.33 & 0.58 & 0.56 & 0.03 & 0.87 \\
\hline$-h=1$ & 0.39 & 0.50 & -1.01 & 0.32 & 0.23 & 0.63 & 0.33 & 0.45 & -1.30 & 0.20 & 0.33 & 0.57 \\
\hline$-h=2$ & 0.56 & 0.75 & -0.88 & 0.38 & 0.64 & 0.42 & 0.37 & 0.65 & -1.56 & 0.12 & 1.05 & 0.30 \\
\hline$-h=3$ & 0.61 & 1.05 & -1.19 & 0.23 & 1.58 & 0.21 & 0.40 & 0.85 & -1.55 & 0.12 & 1.91 & 0.17 \\
\hline$-h=4$ & 0.51 & 1.24 & -1.65 & 0.10 & 1.55 & 0.21 & 0.47 & 1.03 & -1.98 & 0.05 & 1.27 & 0.26 \\
\hline$-\operatorname{VAR}(4)-\mathrm{h}=0$ & 0.48 & 0.44 & 0.69 & 0.49 & 0.92 & 0.34 & 0.42 & 0.44 & -0.48 & 0.63 & 0.74 & 0.39 \\
\hline$-h=1$ & 0.58 & 0.52 & 0.93 & 0.36 & 0.49 & 0.48 & 0.44 & 0.50 & -0.75 & 0.45 & 0.64 & 0.42 \\
\hline$-h=2$ & 0.74 & 0.81 & -0.30 & 0.77 & 0.25 & 0.62 & 0.48 & 0.72 & -1.17 & 0.24 & 1.61 & 0.20 \\
\hline$-h=3$ & 0.88 & 1.10 & -0.53 & 0.60 & 0.50 & 0.48 & 0.52 & 0.94 & -1.11 & 0.27 & 1.89 & 0.17 \\
\hline$-h=4$ & 0.90 & 1.26 & -0.78 & 0.44 & 0.77 & 0.38 & 0.65 & 1.01 & -1.13 & 0.26 & 1.50 & 0.22 \\
\hline \multicolumn{13}{|c|}{ Time-varying gains } \\
\hline$-\operatorname{VAR}(1)-h=0$ & 0.56 & 0.63 & -0.89 & 0.38 & 1.79 & 0.18 & 0.55 & 0.63 & -1.20 & 0.23 & 2.49 & 0.11 \\
\hline$-h=1$ & 0.47 & 0.78 & -1.73 & 0.09 & 0.00 & 0.94 & 0.50 & 0.76 & -1.56 & 0.12 & 0.14 & 0.71 \\
\hline$-h=2$ & 0.33 & 0.89 & -2.58 & 0.01 & 2.92 & 0.09 & 0.35 & 0.81 & -1.86 & 0.07 & 3.18 & 0.07 \\
\hline$-h=3$ & 0.29 & 1.06 & -2.28 & 0.02 & 3.18 & 0.07 & 0.26 & 0.99 & -1.87 & 0.06 & 5.83 & 0.02 \\
\hline$-h=4$ & 0.42 & 1.36 & -1.93 & 0.06 & 2.04 & 0.15 & 0.41 & 1.22 & -1.62 & 0.11 & 2.13 & 0.14 \\
\hline$-\operatorname{VAR}(2)-h=0$ & 0.40 & 0.50 & -1.50 & 0.14 & 0.14 & 0.71 & 0.38 & 0.43 & -1.27 & 0.21 & 2.33 & 0.13 \\
\hline$-h=1$ & 0.41 & 0.72 & -1.99 & 0.05 & 0.01 & 0.94 & 0.46 & 0.64 & -1.42 & 0.16 & 0.08 & 0.77 \\
\hline$-h=2$ & 0.28 & 0.87 & -2.68 & 0.01 & 2.63 & 0.10 & 0.32 & 0.71 & -2.08 & 0.04 & 2.07 & 0.15 \\
\hline$-h=3$ & 0.25 & 1.30 & -2.47 & 0.01 & 7.65 & 0.01 & 0.31 & 1.03 & -2.10 & 0.04 & 5.96 & 0.01 \\
\hline$-h=4$ & 0.37 & 1.52 & -2.24 & 0.03 & 1.64 & 0.20 & 0.47 & 1.28 & -2.07 & 0.04 & 1.41 & 0.23 \\
\hline - $\operatorname{VAR}(3)-h=0$ & 0.36 & 0.38 & -0.24 & 0.81 & 0.73 & 0.39 & 0.31 & 0.33 & -0.37 & 0.71 & 0.00 & 0.98 \\
\hline$-h=1$ & 0.36 & 0.53 & -1.70 & 0.09 & 0.40 & 0.53 & 0.33 & 0.46 & -1.42 & 0.16 & 0.05 & 0.83 \\
\hline$-h=2$ & 0.37 & 0.80 & -2.07 & 0.04 & 0.60 & 0.44 & 0.31 & 0.66 & -1.88 & 0.06 & 1.17 & 0.28 \\
\hline$-h=3$ & 0.34 & 1.08 & -2.15 & 0.03 & 2.12 & 0.14 & 0.33 & 0.87 & -1.84 & 0.07 & 1.87 & 0.17 \\
\hline$-h=4$ & 0.29 & 1.31 & -2.28 & 0.02 & 1.54 & 0.21 & 0.40 & 1.06 & -2.16 & 0.03 & 1.38 & 0.24 \\
\hline$-\operatorname{VAR}(4)-h=0$ & 0.48 & 0.48 & -0.01 & 0.99 & 1.02 & 0.31 & 0.42 & 0.43 & -0.26 & 0.79 & 1.04 & 0.31 \\
\hline$-h=1$ & 0.51 & 0.54 & -0.29 & 0.77 & 0.46 & 0.50 & 0.44 & 0.51 & -0.86 & 0.39 & 0.70 & 0.40 \\
\hline$-h=2$ & 0.58 & 0.83 & -1.23 & 0.22 & 0.02 & 0.90 & 0.43 & 0.74 & -1.50 & 0.14 & 1.41 & 0.24 \\
\hline$-h=3$ & 0.65 & 1.12 & -1.32 & 0.19 & 0.34 & 0.56 & 0.42 & 0.97 & -1.47 & 0.14 & 1.63 & 0.20 \\
\hline$-\mathrm{h}=4$ & 0.45 & 1.26 & -2.00 & 0.05 & 0.79 & 0.37 & 0.45 & 1.01 & -1.84 & 0.07 & 1.52 & 0.22 \\
\hline
\end{tabular}


Table 7: Paired comparisons of algorithms forecasting resemblance for growth.

\begin{tabular}{|c|c|c|c|c|c|c|c|c|c|c|c|c|}
\hline \multirow{3}{*}{$\begin{array}{l}\text { Calibration } \\
\text { - Model } \\
\\
\quad \text { - horizon }\end{array}$} & \multicolumn{6}{|c|}{ Gains as a choice } & \multicolumn{6}{|c|}{ Gains as a primitive } \\
\hline & \multicolumn{2}{|c|}{ MSFCE } & \multicolumn{2}{|c|}{ DM } & \multicolumn{2}{|c|}{ GW } & \multicolumn{2}{|c|}{ MSFCE } & \multicolumn{2}{|c|}{ DM } & \multicolumn{2}{|c|}{ GW } \\
\hline & LS & $S G$ & Stat. & p-val. & Stat. & p-val. & LS & $S G$ & Stat. & $\mathrm{p}$-val. & Stat. & p-val. \\
\hline \multicolumn{13}{|l|}{ Fixed gains } \\
\hline$-\operatorname{VAR}(1)-h=0$ & 2.74 & 2.16 & 1.39 & 0.17 & 2.82 & 0.09 & 2.46 & 2.16 & 0.66 & 0.51 & 3.27 & 0.07 \\
\hline$-h=1$ & 1.56 & 2.39 & -1.68 & 0.10 & 8.44 & 0.00 & 1.35 & 2.27 & -1.73 & 0.09 & 9.88 & 0.00 \\
\hline$-h=2$ & 0.70 & 2.85 & -4.13 & 0.00 & 13.44 & 0.00 & 0.79 & 2.68 & -2.96 & 0.00 & 9.08 & 0.00 \\
\hline$-h=3$ & 0.55 & 3.36 & -5.21 & 0.00 & 15.41 & 0.00 & 0.58 & 2.14 & -1.74 & 0.09 & 3.11 & 0.08 \\
\hline$-\mathrm{h}=4$ & 0.42 & 3.21 & -5.43 & 0.00 & 16.35 & 0.00 & 0.45 & 1.86 & -1.85 & 0.07 & 2.69 & 0.10 \\
\hline$-\operatorname{VAR}(2)-\mathrm{h}=0$ & 2.42 & 2.11 & 0.85 & 0.40 & 3.16 & 0.08 & 2.23 & 2.10 & 0.53 & 0.59 & 3.07 & 0.08 \\
\hline$-h=1$ & 1.43 & 2.02 & -1.43 & 0.15 & 0.40 & 0.53 & 1.28 & 2.00 & -2.22 & 0.03 & 0.46 & 0.50 \\
\hline$-h=2$ & 0.87 & 2.55 & -3.58 & 0.00 & 8.01 & 0.00 & 0.87 & 2.45 & -3.41 & 0.00 & 7.02 & 0.01 \\
\hline$-h=3$ & 0.66 & 3.15 & -4.97 & 0.00 & 15.29 & 0.00 & 0.66 & 2.84 & -4.21 & 0.00 & 10.68 & 0.00 \\
\hline$-h=4$ & 0.48 & 3.15 & -5.35 & 0.00 & 16.82 & 0.00 & 0.48 & 2.83 & -4.30 & 0.00 & 11.19 & 0.00 \\
\hline$-\operatorname{VAR}(3)-h=0$ & 2.59 & 2.12 & 1.19 & 0.24 & 2.17 & 0.14 & 2.59 & 2.11 & 1.22 & 0.23 & 2.17 & 0.14 \\
\hline$-h=1$ & 1.78 & 2.01 & -0.58 & 0.56 & 0.77 & 0.38 & 1.78 & 1.93 & -0.38 & 0.70 & 0.37 & 0.54 \\
\hline$-h=2$ & 1.05 & 2.53 & -2.86 & 0.01 & 4.85 & 0.03 & 1.05 & 2.42 & -2.62 & 0.01 & 4.12 & 0.04 \\
\hline$-h=3$ & 0.85 & 2.89 & -4.02 & 0.00 & 9.91 & 0.00 & 0.85 & 2.81 & -3.53 & 0.00 & 7.89 & 0.00 \\
\hline$-h=4$ & 0.61 & 3.05 & -4.66 & 0.00 & 14.28 & 0.00 & 0.61 & 2.81 & -3.82 & 0.00 & 10.63 & 0.00 \\
\hline$-\operatorname{VAR}(4)-h=0$ & 2.70 & 2.24 & 0.96 & 0.34 & 3.50 & 0.06 & 2.70 & 2.21 & 1.00 & 0.32 & 3.03 & 0.08 \\
\hline$-h=1$ & 2.13 & 1.99 & 0.30 & 0.77 & 1.11 & 0.29 & 1.87 & 1.94 & -0.17 & 0.86 & 0.84 & 0.36 \\
\hline$-h=2$ & 1.32 & 2.46 & -2.18 & 0.03 & 2.97 & 0.08 & 1.32 & 2.32 & -1.80 & 0.07 & 2.49 & 0.11 \\
\hline$-h=3$ & 1.20 & 2.92 & -2.95 & 0.00 & 8.14 & 0.00 & 1.05 & 2.77 & -2.85 & 0.01 & 5.90 & 0.02 \\
\hline$-\mathrm{h}=4$ & 0.74 & 2.79 & -3.97 & 0.00 & 10.40 & 0.00 & 1.00 & 2.76 & -2.49 & 0.01 & 6.71 & 0.01 \\
\hline \multicolumn{13}{|c|}{ Time-varying gains } \\
\hline$-\operatorname{VAR}(1)-h=0$ & 2.61 & 2.23 & 1.27 & 0.21 & 1.17 & 0.28 & 2.34 & 2.20 & 0.49 & 0.63 & 0.59 & 0.44 \\
\hline$-h=1$ & 1.62 & 2.08 & -0.75 & 0.45 & 3.64 & 0.06 & 1.23 & 1.83 & -1.32 & 0.19 & 8.17 & 0.00 \\
\hline$-h=2$ & 1.14 & 2.25 & -1.61 & 0.11 & 7.01 & 0.01 & 0.73 & 2.08 & -2.15 & 0.03 & 6.41 & 0.01 \\
\hline$-h=3$ & 0.64 & 2.59 & -2.91 & 0.00 & 7.49 & 0.01 & 0.54 & 2.36 & -2.44 & 0.02 & 5.68 & 0.02 \\
\hline$-h=4$ & 0.54 & 2.25 & -2.67 & 0.01 & 7.11 & 0.01 & 0.43 & 2.04 & -2.40 & 0.02 & 4.38 & 0.04 \\
\hline$-\operatorname{VAR}(2)-h=0$ & 2.61 & 2.30 & 0.88 & 0.38 & 6.13 & 0.01 & 2.21 & 2.11 & 0.34 & 0.73 & 5.86 & 0.02 \\
\hline$-h=1$ & 1.27 & 2.02 & -1.77 & 0.08 & 3.90 & 0.05 & 1.31 & 1.87 & -1.58 & 0.12 & 2.00 & 0.16 \\
\hline$-h=2$ & 1.78 & 2.36 & -0.55 & 0.59 & 8.78 & 0.00 & 0.88 & 2.11 & -2.62 & 0.01 & 5.58 & 0.02 \\
\hline$-h=3$ & 0.71 & 2.76 & -3.52 & 0.00 & 9.05 & 0.00 & 0.64 & 2.42 & -3.45 & 0.00 & 6.98 & 0.01 \\
\hline$-h=4$ & 1.10 & 2.73 & -1.99 & 0.05 & 4.11 & 0.04 & 0.50 & 2.40 & -3.75 & 0.00 & 7.99 & 0.00 \\
\hline$-\operatorname{VAR}(3)-h=0$ & 2.85 & 2.24 & 1.50 & 0.14 & 0.01 & 0.94 & 2.76 & 2.15 & 1.19 & 0.24 & 1.11 & 0.29 \\
\hline$-h=1$ & 1.83 & 1.97 & -0.28 & 0.78 & 3.81 & 0.05 & 1.84 & 1.87 & -0.06 & 0.95 & 5.71 & 0.02 \\
\hline$-h=2$ & 1.46 & 2.49 & -1.19 & 0.24 & 7.46 & 0.01 & 1.08 & 2.16 & -2.01 & 0.05 & 4.09 & 0.04 \\
\hline$-h=3$ & 1.14 & 2.80 & -2.37 & 0.02 & 6.97 & 0.01 & 0.83 & 2.52 & -2.98 & 0.00 & 5.37 & 0.02 \\
\hline$-h=4$ & 4.85 & 2.76 & 0.50 & 0.62 & 0.50 & 0.48 & 0.61 & 2.47 & -3.37 & 0.00 & 7.84 & 0.01 \\
\hline$-\operatorname{VAR}(4)-h=0$ & 2.87 & 2.36 & 1.17 & 0.24 & 0.02 & 0.88 & 2.82 & 2.21 & 1.10 & 0.27 & 1.48 & 0.22 \\
\hline$-h=1$ & 2.17 & 2.03 & 0.24 & 0.81 & 1.08 & 0.30 & 2.13 & 1.91 & 0.48 & 0.63 & 4.17 & 0.04 \\
\hline$-h=2$ & 1.65 & 2.45 & -1.02 & 0.31 & 5.45 & 0.02 & 1.65 & 2.18 & -0.72 & 0.47 & 2.48 & 0.12 \\
\hline$-h=3$ & 1.41 & 2.89 & -1.95 & 0.05 & 3.40 & 0.07 & 1.39 & 2.55 & -1.46 & 0.15 & 2.87 & 0.09 \\
\hline$-h=4$ & 3.30 & 2.70 & 0.23 & 0.82 & 0.33 & 0.57 & 1.07 & 2.55 & -2.22 & 0.03 & 2.76 & 0.10 \\
\hline
\end{tabular}

See footnotes to table 4 .

We also complement our results in the main text by presenting in tables 8 and 9 the hit 
rates calculated using a significance level of $10 \%$. Clearly, we observe a systematic decrease in these hit rate measures compared to those presented in the main text assuming a $20 \%$ minimum level of significance. This difficulty in detecting statistical differences between the algorithms series of forecasts at lower levels of significance may be a signal of low power of these tests for the comparisons we are conducting.

Table 8: Hit rates, at 10\% significance level, comparing the algorithms forecast accuracy.

\begin{tabular}{|c|c|c|c|c|c|c|}
\hline \multirow{2}{*}{$\begin{array}{l}\text { Variables } \\
\text { - Gains }\end{array}$} & \multicolumn{3}{|c|}{ LS wins } & \multicolumn{3}{|c|}{ SG wins } \\
\hline & Hit rate & DM-10\% & GW-10\% & Hit rate & DM-10\% & GW-10\% \\
\hline \multicolumn{7}{|c|}{ Inflation, gains as a choice } \\
\hline - Fixed & $20 \%$ & $0 \%$ & $0 \%$ & $80 \%$ & $5 \%$ & $10 \%$ \\
\hline - Time-varying & $35 \%$ & $0 \%$ & $0 \%$ & $65 \%$ & $10 \%$ & $5 \%$ \\
\hline \multicolumn{7}{|c|}{ Inflation, gains as a primitive } \\
\hline - Fixed & $65 \%$ & $0 \%$ & $0 \%$ & $35 \%$ & $15 \%$ & $10 \%$ \\
\hline - Time-varying & $60 \%$ & $0 \%$ & $0 \%$ & $40 \%$ & $5 \%$ & $10 \%$ \\
\hline \multicolumn{7}{|c|}{ Growth, gains as a choice } \\
\hline - Fixed & $95 \%$ & $50 \%$ & $0 \%$ & $5 \%$ & $0 \%$ & $0 \%$ \\
\hline - Time-varying & $75 \%$ & $10 \%$ & $10 \%$ & $25 \%$ & $0 \%$ & $5 \%$ \\
\hline \multicolumn{7}{|c|}{ Growth, gains as a primitive } \\
\hline - Fixed & $100 \%$ & $45 \%$ & $0 \%$ & $0 \%$ & $0 \%$ & $0 \%$ \\
\hline - Time-varying & $100 \%$ & $40 \%$ & $10 \%$ & $0 \%$ & $0 \%$ & $0 \%$ \\
\hline
\end{tabular}

See the main text for explanations.

Table 9: Hit rates, at $10 \%$ significance level, comparing the algorithms forecast resemblance to surveys.

\begin{tabular}{|c|c|c|c|c|c|c|}
\hline \multirow{2}{*}{$\begin{array}{l}\text { Variables } \\
\text { - Calibrations }\end{array}$} & \multicolumn{3}{|c|}{ LS wins } & \multicolumn{3}{|c|}{ SG wins } \\
\hline & Hit rate & DM-10\% & GW-10\% & Hit rate & DM-10\% & GW-10\% \\
\hline \multicolumn{7}{|c|}{ Inflation, gains as a choice } \\
\hline - Fixed & $80 \%$ & $30 \%$ & $10 \%$ & $20 \%$ & $5 \%$ & $0 \%$ \\
\hline - Time-varying & $100 \%$ & $65 \%$ & $15 \%$ & $0 \%$ & $0 \%$ & $0 \%$ \\
\hline \multicolumn{7}{|c|}{ Inflation, gains as a primitive } \\
\hline - Fixed & $95 \%$ & $20 \%$ & $15 \%$ & $5 \%$ & $0 \%$ & $0 \%$ \\
\hline - Time-varying & $100 \%$ & $45 \%$ & $15 \%$ & $0 \%$ & $0 \%$ & $0 \%$ \\
\hline \multicolumn{7}{|c|}{ Growth, gains as a choice } \\
\hline - Fixed & $75 \%$ & $65 \%$ & $65 \%$ & $25 \%$ & $0 \%$ & $15 \%$ \\
\hline - Time-varying & $65 \%$ & $35 \%$ & $65 \%$ & $35 \%$ & $0 \%$ & $5 \%$ \\
\hline \multicolumn{7}{|c|}{ Growth, gains as a primitive } \\
\hline - Fixed & $80 \%$ & $70 \%$ & $55 \%$ & $20 \%$ & $0 \%$ & $15 \%$ \\
\hline - Time-varying & $75 \%$ & $50 \%$ & $65 \%$ & $25 \%$ & $0 \%$ & $10 \%$ \\
\hline
\end{tabular}




\section{References}

Barucci, E., Landi, L., 1997. Least mean squares learning in self-referential linear stochastic models. Economics Letters 57, 313-317.

Benveniste, A., Metivier, M., Priouret, P., 1990. Adaptive Algorithms and Stochastic Approximations. Springer-Verlag.

Berardi, M., Galimberti, J.K., 2012. On the initialization of adaptive learning algorithms: A review of methods and a new smoothing-based routine. Centre for Growth and Business Cycle Research Discussion Paper Series 175. Economics, The Univeristy of Manchester.

Berardi, M., Galimberti, J.K., 2014. On the determination of adaptive learning gains. Mimeo.

Branch, W.A., Evans, G.W., 2006. A simple recursive forecasting model. Economics Letters $91,158-166$.

Bullard, J., Eusepi, S., 2005. Did the great inflation occur despite policymaker commitment to a taylor rule? Review of Economic Dynamics 8, $324-359$.

Carceles-Poveda, E., Giannitsarou, C., 2007. Adaptive learning in practice. Journal of Economic Dynamics and Control 31, 2659-2697.

Diebold, F.X., Mariano, R.S., 1995. Comparing predictive accuracy. Journal of Business \& Economic Statistics 13, 253-63.

Evans, G.W., Honkapohja, S., 1998. Stochastic gradient learning in the cobweb model. Economics Letters 61, 333-337.

Evans, G.W., Honkapohja, S., 2001. Learning and expectations in macroeconomics. Frontiers of Economic Research, Princeton University Press, Princeton, NJ.

Faust, J., Wright, J.H., 2013. Chapter 1 - forecasting inflation, in: Elliott, G., Timmermann, A. (Eds.), Handbook of Economic Forecasting. Elsevier. volume 2, Part A of Handbook of Economic Forecasting, pp. 2 - 56.

Giacomini, R., White, H., 2006. Tests of conditional predictive ability. Econometrica 74, 15451578. 
Giannitsarou, C., 2005. E-stability does not imply learnability. Macroeconomic Dynamics 9, 276-287.

Heinemann, M., 2000. Convergence of adaptive learning and expectational stability: The case of multiple rational-expectations equilibria. Macroeconomic Dynamics 4, 263-288.

Markiewicz, A., Pick, A., 2014. Adaptive learning and survey data. DNB Working Papers 411. Netherlands Central Bank, Research Department.

Newey, W.K., West, K.D., 1987. A simple, positive semi-definite, heteroskedasticity and autocorrelation consistent covariance matrix. Econometrica 55, 703-08.

Patton, A.J., Timmermann, A., 2011. Predictability of output growth and inflation: A multihorizon survey approach. Journal of Business and Economic Statistics 29, 397-410.

Stark, T., Croushore, D., 2002. Forecasting with a real-time data set for macroeconomists. Journal of Macroeconomics 24, $507-531$.

Stock, J.H., Watson, M.W., 2003. Has the business cycle changed and why?, in: NBER Macroeconomics Annual 2002, Volume 17. National Bureau of Economic Research, Inc. NBER Chapters, pp. 159-230. 